\title{
Anesthetic Management of a Patient with Brugada Syndrome - the Use of Sugammadex in Major Abdominal Surgery
}

Dear Editor,

Brugada Syndrome (BS) is a rare genetic condition affecting ionic channels of the heart, predisposing patients to potentially fatal ventricular arrhythmias. It generally manifests during young adulthood, more frequently in males. Typical ECG pattern shows ST-segment elevation followed by a negative T wave in right pre-cordial leads, in the absence of structural heart disease. Three patterns can be recognized: 1) coved; 2) saddleback and 3) coved or saddleback with $\mathrm{ST}<1 \mathrm{~mm}$. These can co-exist and ECG may be temporarily normal. Diagnosis is made in the presence of ECG pattern type 1 and reinforced by clinical criteria like syncope, nocturnal agonal respiration, palpitations or dizziness, documented ventricular fibrillation/tachycardia and family history of BS or sudden cardiac death (SCD). Provocative tests can be made with sodium channel blockers. Cardiac defibrillator (ICD) implantation is recommended for all symptomatic patients and for those who, though asymptomatic, present inducible VT/VF during electrophysiologic study (EPS). Quinidine can be used safely as anti-arrhythmic agent when ICD implantation is not possible ${ }^{1}$.

\section{Case Report}

A 67-yr-old male was diagnosed with colon cancer and scheduled for a sub-total colectomy. Six years before, he had an ICD (VVIR) implanted after asymptomatic type I Brugada syndrome was diagnosed based on typical ECG pattern, easily inducible VT on EPS. Before surgery, ICD was externally disabled to prevent inadequate activation by monopolar cautery; from then on an external defibrillator being permanently available. After premedication with midazolam $(1 \mathrm{mg})$, an epidural thoracic catheter was placed. Induction was achieved with fentanyl $\left(0.02 \mathrm{mg} \cdot \mathrm{kg}^{-1}\right)$ and propofol $\left(2 \mathrm{mg} \mathrm{kg}^{-1}\right)$. Orotracheal intubation was performed uneventfully after neuromuscular blockade with rocuronium $\left(0.6 \mathrm{mg} . \mathrm{kg}^{-1}\right)$. Anesthesia was maintained with sevoflurane, rocuronium and epidural fentanyl. Monitorization included 5-lead ECG and ST trend analysis, invasive arterial blood pressure, peripheral arterial oxygen saturation, end tidal $\mathrm{CO}_{2}$, esophageal temperature, $\mathrm{BIS}$, neuromuscular blockade and urine output. No dysrhythmia or ST-segment elevations were noted during the entire procedure, which lasted approximately 2 hours. Neuromuscular blockade was reversed with sugammadex $\left(2 \mathrm{mg} \cdot \mathrm{kg}^{-1}\right)$ and the patient extubated without incidents. Analgesia was provided with epidural ropivacaine and morphine. In the post anesthetic care unit, ICD was re-activated. After 6 days the patient was discharged home, with no adverse events being reported.

\section{Discussion}

In BS patients, several pharmacological and physiological factors can initiate malignant arrhythmias, including medications routinely used in anaesthetic practice, electrolyte disturbances, temperature variations, physiologic stress and increased vagal activity, all of which must be kept in the mind of the anesthetist managing these patients ${ }^{2,3}$.

On this subject, recommendations are sparse and limited by conflicting results found in literature, probably due to inter individual variability, medication associations and doses and physiologic circumstances ${ }^{4}$. Propofol has been associated with the development of ventricular arrhythmias in prolonged infusions, suggesting a possible similar mechanism to that responsible for arrhythmogenesis in propofol infusion syndrome, rather than with bolus dosing for induction ${ }^{5}$. Local anesthetics - such as class Ib anti-arrhythmic agents and block sodium channels - should raise concern to the anesthesiologist. Though, when used cautiously, with the dose minimized and close monitoring of the patient, regional techniques may be helpful in avoiding light anesthesia and analgesia, factors known to affect autonomic tone. Particular attention should be paid to bupivacaine which stays bound to the sodium channels for longer periods of time and causes greater depression of rapid phase depolarization in the ventricular 


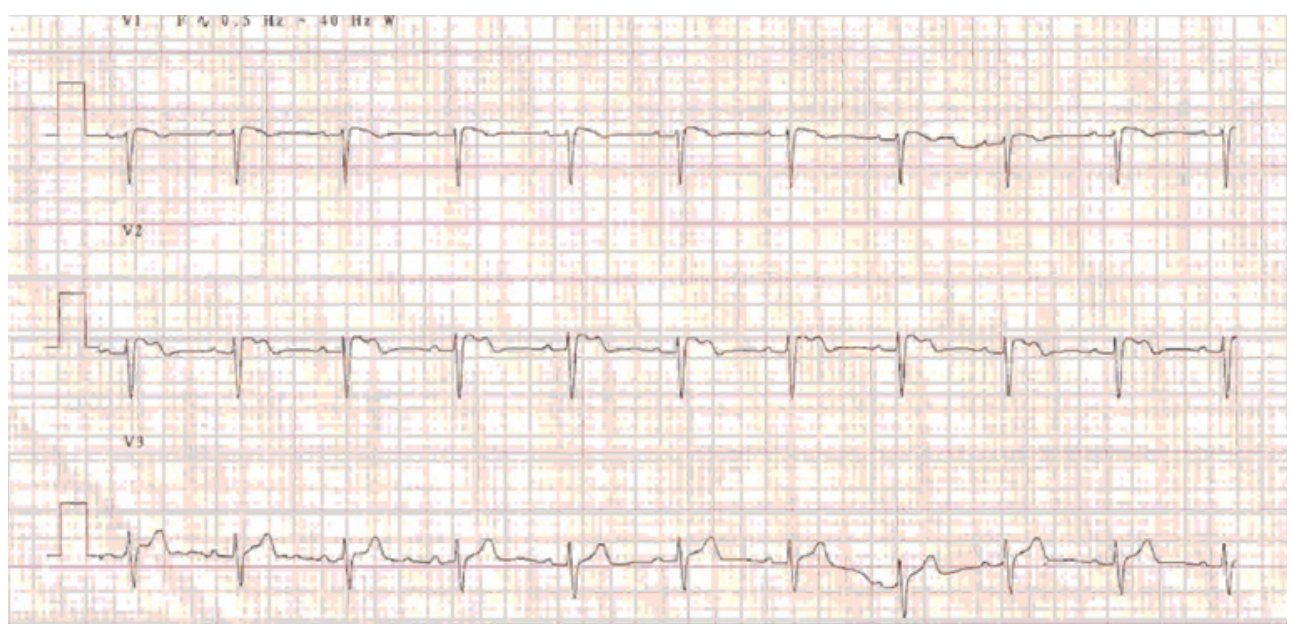

Figure 1 Patient ECG, Showing Type II (saddleback) Pattern in V2.

muscle. Regarding volatile anesthetics, preference is given to sevoflurane as it provides a better stability of QT interval. Though it has been safely used ${ }^{6}$, neostigmine is best avoided in the reversal of neuromuscular blockade as it may augment ST segment elevation ${ }^{7}$.

In this case we preferred to use sugammadex, a $\gamma$-cyclodextrine with a lipophylic center that encapsulates rocuronium (and vecuronium) molecules forming water soluble complexes that then are excreted in urine. Thereby, rapid and effective reversal of neuromuscular blockade is achieved, decreasing the risk of residual paralysis, particularly relevant in the case of major abdominal surgery, avoiding the side effects associated with neostigmine and anti-muscarinic agents, notably significant in BS patients.

\section{Conclusions}

BS is a rare though potentially fatal condition. Threatening arrhythmias can be triggered by several factors often present during surgical procedures, putting patient safety at stake. Most importantly, in the anesthetic management of BS patients, the decision of using each drug must be made after extensive consideration and in controlled conditions, avoiding other factors that are known to have the potential to induce arrhythmias like electrolyte and temperature disturbances, maintaining a close monitoring on hemodynamic status of the patient and being prepared to readily interfere if such events occur. Regarding the reversal of neuromuscular blockade, suggamadex presents as a safer option in BS patients, granting the desired goal while avoiding undesirable cardiovascular and autonomic effects.

\section{Rita Conde ${ }^{1}$, Marta Pereira ${ }^{2}$ 1. Residency, \\ 2. Anesthesiology Expert} Anaesthesiology and Pain Treatment Department, Centro Hospitalar de Trás-os-Montes e Alto Douro, Portugal

\section{References}

1. Benito B, Brugada J, Brugada R et al. - Brugada Syndrome. Rev Esp Cardiol, 2009;62(11):1297-1315.

2. Carey SM, Hocking G. - Brugada Syndrome - a review of the implications for the anaesthetist. Anaesth Intensive Care, 2011;39:571-577.

3. Kloesel B, Ackerman MJ, Sprung J et al. - Anesthetic management of patients with Brugada syndrome: a case series and literature review. Can J Anesth 2011;58:824-836.

4. Postema PG, Wolpert C, Amin AS et al. - Drugs and Brugada syndrome patients: review of the literature, recommendations and an up-to-date website. Heart Rhythm, 2009;6(9):13351341.

5. Yap YG, Behr ER, Camm AJ - Drug induced Brugada syndrome. Europace, 2009;11:989-994.

6. Hayashida $\mathrm{H}$, Miyauchi $\mathrm{Y}$ - Anaesthetic management in patients with high-risk Brugada syndrome. Br J of Anaesth, 2006;97(1):118119.

7. Edge CJ, Blackman DJ, Gupta K et al. - General anesthesia in a patient with Brugada syndrome. Br J of Anaesth, 2002;89:788791. 